\title{
Ueber einen Fall \\ von Luxation der Linse in den Tenon'schen Raum bei äquatorial gelegenem Skleralriss.
}

\author{
Von \\ Dr. med. Walter Schlodtmann, \\ Assistenten an der Königl. Universitäts-Augenklinik \\ zu Halle a. S. \\ Mit Tafel VI, Fig. 1 und 2.
}

Die Prädilectionsstelle für indirecte Berstungen der Corneaskleralkapsel durch stumpfe Gewalt ist bekanntlich die Corneoskleralgrenze selbst, resp. eine schmale Zone der Sklera unmittelbar hinter dem Limbus. Michel ${ }^{1}$ ) bezeichnet diejenigen Fälle, bei denen der Riss 2-4 mm hinter dem Limbus liegt, bereits als selten. Kommt es bei einer solchen Verletzung zu einer Luxation der Linse durch den Skleralriss, ohne dass die Conjunctiva mit zerreisst, so gleitet die Linse, falls die Luxation eine vollständige ist, durch die Wunde unter die Conjunctiva und bleibt in der Nähe der Austrittsstelle, also im vorderen Bulbusabschnitte, liegen. Nur ganz vereinzelt sind bisher Fälle beobachtet worden, bei denen der Riss der Sklera im Aequator oder gar im linteren Bulbusabschnitte lag. Müller ${ }^{2}$ ) erwähnt in seiner sehr ausfiuhrlichen Arbeit über Skleralrupturen, dass bisher

1) Lehrbuch der Angenheilkunde, II. Auflage 1890, S. 669.

2) Ueber Ruptur der Corneoskleralkapsel durch stumpfe Verletzung, Leipzig u. Wien 1895, S. 18. 
nur vier solcher Fälle bekannt seien, nämlich von $\mathrm{Nuel}$, Weeks, Chisolm und Bowman. Eine gleiche Seltenheit ist es, wenn die Linse, sei es in Folge der Lage des Risses, sei es in Folge bestimmter mechanischer Momente, soweit nach hinten gelangt, dass sie am hinteren Bulbusabschnitte, in den Tenon'schen Raum zu liegen kommt. In der Titeratur finde ich nur drei derartige Fälle erwähnt, wovon einer nicht einmal eine vollständige Luxation in den Tenon'schen Raum, sondern eine halb subconjunctivale Luxation darstellt, ein zweiter uiberhaupt jeder detaillirten Angabe entbehrt.

$\mathrm{Da}$ in keinem derselben eine anatomische Untersuchung vorgenommen ist, möchte ich im Nachstehenden eine Schilderung, hauptsächlich der histologischen Verhältnisse geben, wie sie sich an einem Bulbus mit äquatorial gelegener Skleralruptur und vollständiger Luxation der Linse in den Tenon'schen Raum vorfanden.

Für die Ueberlassung des Falles zur Bearbeitung sowie für die Anregung dazu sage ich meinem hochverehrten Chef, Herrn Geheimrath v. Hippel, meinen aufrichtigsten Dank.

Die Krankengeschichte ist in Kürze folgende:

Anamnese: Als Patient, der 66 Jahre alte Arbeiter A. B., am Tage seiner Aufnahme in die Klinik, Mittags ein Ochsenfuhrwerk anspannen wollte, stiess ihm eins der Thiere sein Horn in das linke Auge. Ueber die näheren Ümstände, wie Richtủng des Stosses, augenblickliche Kopfhaltung etc., konnte nichts Genaueres eruirt werden. Patient verspürte alsbald einen heftigen Schmerz und konnte auf dem linken Auge nichts mehr sehen. Der consultirte Arzt ordnete die sofortige Ueberführung nach Halle an, wo Patient noch an demselben Abend in die Klinik aufgenommen wurde.

Status praesens vom 25. IV. 96: R. A. normal ES $=1$. L. A. Partielle Zerreissung des oberen Lides auf der Grenze zwischen seinem mittleren und äusseren Dritttheil. Starke Lichtscheu, Epiphora, conjunctivale Injection. Conjunctiva bulbi hochgradig chemotisch. Cornea klar, Vorderkammer völlig vou einem 
Ein Fall von Luxation der Linse in den Tenon'schen Raum etc. 129

starken Bluterguss eingenommen, der jeden Einblick in das Augeninnere verhindert. Der ausserordentlich herabgesetzte Tonus deutet auf eine Ruptur der Sklera, von welcher sich jedoch zur Zeit direct nichts nachweisen lässt.

Ordo: Lidsuturen, Verband, Bettruhe.

26. IV. - 4. V. Allmähliche Resorption des Blutes in der Vorderkammer bis auf ein minimales Hyphaema. Ebenso geht die Chemose zurück. Injection noch sehr intensiv. Patient zählt Finger in unmittelbarer Nähe.

5. V. Grosse frische Blutung in die Vorderkammer.

6.-8. V. Langsame Resorption des Blutes. Zeitweiliges Auftreten von Schmerzen. Corpus ciliare nicht druckempfindlieh. Tonus etwas besser.

9. 12. 16. V. Wiederholte frische Blutungen, welche sich schliesslich fast gar nicht mehr resorbiren. Es besteht leichte Druckempfinđlichkeit des Corpus ciliare.

20. V. Punction der Vorderkammer.

25.-29. V. Es treten typische glankomatöse Schmerzen auf, welche an Intensität zunehmen.

29. V. Enucleation in Chloroformnarcose.

4. VI. Entlassung.

Schon während der Enucleation fiel eine halb kugelige tumorartige Erhebung am hinteren Abschnitte des Bulbus anf, deren Verletzung sorgfältig vermieden wurde. Die hohe Consistenz, die Form, sowie der relativ hohe Grad von Transparenz des Tumors deutete darauf hin, dass es sich um die Linse handelte, welche auffallend weit nach hinten luxirt war. Dieselbe lag: zwischen dem M. obliquus superior und M. rectus externus so, dass ihr linterer Rand ungefähr ebenso weit lateral von dem hinteren Bulbuspol entfernt lag, wie der Sehnerv nasal von ihm. Der untere Rand des Tumors lag etwa im horizontalen Meridian, sein vorderer Rand noch hinter dem frontalen Aequator. Der Skleralriss war nicht mit Deutlichkeit zu sehen, jedenfalls nicht scharf abzugrenzen, was sich leicht aus der sehr schrägen Richtung, in welcher er die Sklera durchsetzt, erklärt.

Der enucleirte Bulbus wurde in $4 \%$ Formollösung fixirt und gehärtet und darauf einer Nachbärtung in Alkohol von allmählich gesteigerter Concentration unterworfen. Nach Halbirung durch einen unterhalb des Sehnerven gelegten horizontalen Schnitt wurde er in Celloidin gebettet.

Auf dem Durchsehnitt bot der Bulbus folgendes makroskopische Bild dar: Die Sklera ist überall ron normaler Dicke. 
Etwas hinter der Insertion des M. rectus externus wird sie von einer feinen, dunkeln Linie durchzogen, welche schräg von vorn innen nach hinten aussen verläuft. An dieser Stelle ist die Sklera glaskörperwärts eingezogen, wäbrend sich aussen eine Verdickung findet, die Peripherie des durch die Linse gebildeten Tumors. Unmittelbar hinter dieser Stelle ist eine frische Blutung ins episklerale Zellgewebe sichtbar. Nach innen von dieser wieder befindet sich eine ziemlich ausgedehnte, flache Blutung in der Suprachorioidea, dureh welche also eine Abhebung der Aderhaut bedingt wird. Die Iris ist etwas zurückgezogen, so dass die vordere Augenkammer vertieft erscheint. Die Netzhaut ist vollständig auf der nasalen Seite bis zur Iriswurzel, auf der temporalen bis zur Skleralruptur - abgehoben und liegt vielfach gefaltet im Glaskörper. Auf der Netzhaut liegen zahlreiche ausgedehnte Blutcoagula, welche die Falten der abgehobenen Retina zum Theil vollständig ausfüllen.

Im Bereich der Cornea wurde der Bulbus in horizontale Serienschnitte zerlegt; die übrig bleibenden Segmente wuxden nach verschiedenen Richtungen geschnitten und untersucht. Die mikroskopische Untersuchung ergab folgende Resultate:

Die Hornhaut (Fig. $1 c$ ) ist in ihren mittleren Parthieen von völlig normalem Bau. Erst gegen die Peripherie zu zeigt sie pathologische Veränderungen, welche besonders ausgesprochen nach der temporalen Seite, also der Seite der Verletzung hin, sind. Hier ist das Epithel stark verdickt und sendet in das darunter liegende Gewebe zapfenartige Vorsprünge, welche, wie man an peripheren Schnitten sehen kann, vielfach als radiäre Leisten angeordnet sind. Die Bowman'sche Membran ist eine Strecke weit unterbrochen, zeigt in ihren äussersten Randparthieen eine unregelmässige Structur und hört ein beträchtliches Stïck früher als normal auf. Das Hornhautparenchym betreffend ist zunächst die Punctionsnarbe in der unteren Corneahälfte zn erwälnen, welche nahe der Oberfläche völlig mit Epithelzellen ansgefüllt ist, mehr in der Tiefe dagegen nur vereinzelte isolirte Epithelzellen aufweist und im Uebrigen hier von Bindegewebe gebildet wird. Sonst zeigt das Hornhautparenchym nur in der Peripherie ein von der Norm abweichendes Bild, indem sich, wie man es zuweilen bei länger bestehenden entzündlichen Affectionen sieht, das von zahlreichen, meist mononucleären Zellen infiltrirte und von einer Menge strotzend gefüllter Gefässe durchsetzte episklerale Gewebe über die Corneoskleralgrenze hinaus zwischen Hornhautparenchym und -epithel vorschiebt und hier 
gewissermaassen noch ein epicorneales Infiltrationsgewebe bildet. Die stark gefüllten Blutgefässe haben an verschiedenen Stellen $\mathrm{zu}$ diffusen Blutungen ins Gewebe geführt, wobei die Blutkörperchen theils in grösseren zusammenhängenden Haufen liegen, theils sich perlschnurartig aneinander reihen und so die Sklera resp. Cornea lamelliren. Nach hinten setzt sich dieses Infiltrationsgewebe, allmählich an Mächtigkeit abnehmend, bis zu der luxirten Linse fort. Die Descemet'sche Haut sowie das sie bedeckende Endothel sind normal; auf letzterem finden sich Auflagerungen von rothen Blutkörperchen, welche peripherwärts an Zahl zunehmen, so dass der Kammerwinkel völlig von Blut erfüllt erscheint.

Aehnlich prall gefüllte Blutgefässe, wie sie die Episklera zeigt, durchsetzen im Limbus die ganze Dicke der Corneosklera und zeigen hier eine infiltrirte Matrix; sie finden sich ferner in grosser Zahl in der Iris, dem Corpus ciliare, sowohl dem Muskel als den Ciliarfortsätzen, und in der Chorioidea.

Die Iris (Fig. 1, $i$ ) ist an der Seite der Verletzung mit ihrem den Pupillarrand bildenden Theil nach hinten umgeschlagen. Die Iriswurzel ist an einzelnen Stellen auffallend dünn and atrophisch. In der Nähe des Pupillarrandes sind stellenweise kleine Blutungen ins Gewebe erfolgt.

Am Corpus ciliare finden sich einige wenige blasenförmige Epithelabhebungen wie sie Greff ${ }^{1}$ ) als Folgeerscheinungen nach Punction der vorderen Kammer beschrieben hat. Einzelne der Blasen sind von beträchtlicher Grösse und schon makroskopisch wahrnehmbar. So fand ich den Durehmesser des Innenraumes einer der grössten Blasen gleich $696,6 \mu$, also nahezu $3 / 4 \mathrm{~mm}$ lang. Auch hier bestätigt sich die Angabe Greff's, dass bald nur die oberste Epithellage, bald das gesammte Epithel einschliesslich der Pigmentschichte abgehoben sei. Ebenso entspricht die Localisation den bisherigen Beobachtungen: die Blasen sitzen an den vordersten Ciliarfortsätzen, während mehr nach hinten zu keine mehr aufzufinden ist. Zerreissungen lassen sich nirgends im Gewebe des Ciliarkörpers constatiren. Dagegen ist das Corpus ciliare sowohl als der vordere Abschnitt der Chorioidea rings um die ganze Peripherie des Bulbus durch eine Exsudatschicht von wechselnder Dicke abgehoben. Am mächtigsten ist sie überall in der Gegend der Ora serrata; von hier sehiebt sie sich nach vorn

1) Befund am Corpus ciliare nach Punction der vorderen Kammer. Archiv für Augenheilkunde. 1894, Bd. 28, S. 178-192. 
und hinten keilförmig zwischen Sklera einerseits und Corpus ciliare resp. Chorioidea andererseits vor bis zu deren normalem Contact. Nur in der Gegend des Skleralrisses verjüngt sich diese Exsudatsehichte nicht allmählich, sondern bört hinten, von der eingebogenen Sklera begrenzt, ziemlich plötzilich auf. Hier ist auch die Abhebung der Chorioidea am ausgiebigsten erfolgt, und letztere spannt sich vom Ciliarkörper aus hinüber bis zum hinteren Wundrand, wo sie sich gleich wieder unmittelbar an die Sklera ansetzt und ihr, freilich nicht glatt, sondern vielfach gewellt, direct anliegt. In dem vom Exsudate erfüllten gewölbeartigen Raum spannen sich an verschiedenen Stellen einzelne Bündel des an der Sklera haften gebliebenen Suprachorioidealgewebes zwischen den beiden getrennten Membranen aus, indem sie sie brïckenartig verbinden. Das Pigmentepithel, welches das Corpus ciliare und die vordersten Theile der Chorioidea noch in normaler Weise bekleidet, fehlt an den der Narbe benachbarten Parthieen fast gänzlich; nur spärliche Pigmentballen markiren seinen ursprünglichen Sitz. Im unteren äusseren Bulbusquadranten hat sich eine suprachorioideale, ziemlich ausgedehnte, aber flache Blutung etablirt. Sie reicht nach oben bis etwa zum unteren Rande der luxirten Linse, nach unten .bis fast an den unteren Bulbuspol, nach hinten bis an den sagittalen Bulbusmeridian. An ihrer oberen Grenze geht sie in eine ödematöse Durchtränkung und Quellung der Suprachorioidea ïber. Sonst zeigt die Chorioidea, abgesehen von vereinzelten circumscripten Infiltraten und diffusen Blutungen in das Suprachorioidealgewebe nichts Auffälliges.

Die Netzhaut (Fig. 1, r) ist an der medialen Seite kurz vor der Ora serrata abgelöst und zieht, im rechten Winkel zu ihr, in den Glasskörper, wo sie sich in vielfache Falten gelegt hat. In der Gegend der Verletzung ist die Retina kurz vor der Ora serrata abgerissen. Der Rand des abgerissenen Stüekes setzt sich an die hintere Wundlippe der Sklera an. Da der Sporn der Ora serrata hier nirgends nachweisbar ist, so muss ein Stück aus der Retina herausgerissen sein, worauf ich noch bei Besprechung der topographischen Verhältnisse an der Skleranarbe znrück komme. Nach hinten reicht die Amotio retinae bis direct an die Papilla optica. Der abgehobenen Netzhaut aufliegend und die Zwischenräume zwischen ihren Falten zum Theil vollständig ausfüllend, finden sich massenhafte Anhäufungen von rothen Blutkörperchen, welche einen beträchtlichen Theil des Glaskörperraumes einnehmen. 
In der Nähe der Papille weist die Netzhaut in ihrer äusseren reticulären Sehicht zahlreiche aneinander gereihte eystoide Hohlräume auf, welche durch die feinen Bälkchen der Stützsubstanz von einander getrennt sind. Die genannte Schicht wird dadurch derart verbreitert, dass sie an den betreffenden Stellen etwa die doppelte Dicke jeder der Körnerschichten erreicht. Im Uebrigen ist die Retina, abgesehen von dem keineswegs seltenen Befunde cystoider Degeneration im Sporn der Ora serrata, in ihrer Structur sehr gut exhalten und die einzelnen Schichten derselben überall mit grosser Deutlichkeit zu erkennen. Die mit Ferrocyankalium und Salzsäure behandelten Schnitte zeigen die Perls'sche Eisenreaction, wie sie E. v. Hippel ${ }^{1}$ ) als charakteristisch bei intraoculären Blutungen ohne Anwesenheit eines Eisensplitters im Ange beschrieben hat. Und zwar tritt hier die Reaction, ähnlich wie dort in Fall XIV und $\mathrm{XV}^{2}$ ), auf in Form einer diffusen Blaufärbung des Pigmentepithels der Pars ciliaris retinae, welche sich nach hinten genau bis zur Ora serrata erstreckt, während an der eigentlichen Retina selbst keine Blaufärbung zu constatiren ist.

Der Sehnerv verhält sich normal.

Wenden wir uns nun zur Betrachtung des Skleralrisses und der luxirten Linse (Fig. $1 n$ u. $l$ ), so möchte ich mich bei der Beschreibung zunächst an das Bild halten, wie es sich in einem ungefähr durch die Mitte der Linse gelegten Horizontalschnitte präsentirt. Die vordere Wundlippe zeigt hier eine etwa bajonettförmige Verbiegung, indem die Sklerafasern, wenn man sie von vorn nach hinten verfolgt, zunächst rechtwinklig gegen den Glaskörper eingeknickt sind, der äusserste Rand der Wundlippe aber von dem eingeknickten stück wieder im rechten Winkel nach hinten biegend, der ursprünglichen Richtung der Sklera parallel verläuft (cf. Fig. 1, $a$; in Fig. 2 ist die Einknickung nicht mehr so prononcirt). Die Episklera (Fig. 2,e) macht diesen Verlauf nicht mit, sondern zieht in sanftem Bogen nach hinten zur luxirten Linse, welche sie als Tenon'sche Kapsel iiberkleidet. Den allmählichen Uebergang von dem relativ geraden Verlauf der Episklera bilden die oberflächlichsten, aus dem festen Verbande des skleralen Gewebes gelockerten Sklerafasern,

1) Ueber Siderosis bulbi und die Beziehungen zwischen siderotischer und haematogener Pigmentbildung. v. Graefe's Archiv f. Ophthalm. XI. 1. S. 123-279.

2) Loc. cit. S. 194-195. 
welche nach hinten bis an den vorderen Rand der Linse herantreten, deren Ueberkleidung sie ganz vorn noch bilden helfen. Die zwischen ihnen gelegenen Zwischenräume und Spalten füllt ein kernreiches, hier und da Pigment aufweisendes Bindegewebe von unregelmässiger Structur ans.

Die hintere, nicht so scharf eingeknickte Wundlippe legt sich von innen an die vordere an und ragt nach vorn noch ein wenig über sie hinaus, so dass sie also gleichsam unter die vordere Wundlippe hinunter und vorgeschoben erseheint (cf. Fig. 1, b). In den mittleren Parthieen der Wunde wird das zwischen die beiden Sklerablätter eingelagerte Narben- oder, wie Krü ckman ${ }^{1}$ ) es bezeichnet, "Ersatzgewebe" von einer dünnen Schicht kernreichen Bindegewebes gebildet, das hier ausser etwas Pigment keine fremden Bestandtheile in sich schliesst. Vorn an der inneren Wundöffnung und hinten nach der luxirten Linse zu weichen die Wundlippen ein wenig mehr auseinander, das Narbengewebe verbreitert sich dem entsprechend. An der inneren Mündung des Wundcanals sind noch Theile der Chorioidea in das neugebildete Gewebe eingelagert, die vielfach gesehlängelte Lamina elastica lässt sich sogar noch ein beträchtliches Stück in die Narbe hinein verfolgen. Die nach hinten $\mathrm{zu}$ an die Linse grenzende Parthie des Zwischengewebes ist weniger straff als die mittleren Theile und stellt ein mehr lockeres, sehr gefässreiches Bindegewebe dar.

In den weiter nach oben gelegten Schnitten sind die Wundränder nicht mehr gegen einander verschoben, sondern weichen ziemlich weit auseinander. In dem durch unregelmässig angeordnete Bindegewebszüge ausgefüllten Anfangstheile der Narbe liegt ein rundliches, im Durchmesser etwa $0,7 \mathrm{~mm}$ haltendes Stïck Retina eingesprengt (Fig. 2, r), welches einen Zusammenlaang mit der intrabulbär gelegenen Netzhaut in keinem Schnitte erkennen lässt, also völlig isolirt ljegt. Es enthält neben den relativ gut erhaltenen Netzhantelemente eine beträchtliche Menge rother Blutkörperchen und etwas Uvealpigment. Umgeben ist das Ganze von einer straffen bindegewebigen Kapsel, an welche von vorn und aussen die Sklerafasern direct herantreten; an den übrigen Seiten wird sie von Narbengewebe begrenzt. Fast unmittelbar hinter diesem Retinastück schliessen sich die Sklerablätter wieder dicht aneinander.

1) Experimentelfe Untersuchungen über die Heilungsvorgänge von Lederhantwunden. v. Graefe's Archiv f. Ophthalm. XLII. 4. S. 321 . 
Ein Fall von Luxation der Linse in den Tenon'schen Raum etc. 135

Gegen das obere Ende der Ruptur zu rücken die Wundlippen allmählich ganz nahe aneinander, ohne durch eine nennenswerthe Schicht von Zwischensubstanz getrennt zu werden; die Continuitätstrennung betrifft nur noch die innersten Skleralagen und hört endlich ganz auf, so dass hier nur noeh der von der Norm abweichende bogenförmige Verlauf der Fasern die Stelle bezeichnet, welche der Fortsetzung des Risses entsprechen würde.

Nach unten von der Mitte der Linse hört die Verschiebung der Wundränder gegen einander ebenfalls bald auf, so dass sie also nur auf eine ganz kurze Strecke beschränlst ist. Die Wundlippen sind hier nach innen eingebogen und durch eine narbige Zwischenschicht von beträchtlicher Breite getrennt. In den Anfangstheil der Narbe schiebt sich der Glaskörper ein; die Retina tritt nicht mehr bis an das hintere Sklerablatt heran, sondern ilır abgerissener Rand endigt frei in Glaskörper.

Unterhalb des horizontalen Bulbusäquators lässt sich der Sklerariss als solcher nicht mehr weit verfolgen. Er betrifft bald nur noch die innersten Skleralagen, verläuft dann ein Stück zwischen inneren und mittleren Sklerafasern nach hinten und geht unmerklich in eine Linie über, an welcher kein neugebildetes Zwischengewebe mehr zu constatiren ist, welche aber - ähnlich, wie an der oberen Grenze der Ruptur - doch erkennen lässt, dass hier noch eine Zerrung im Skleragewebe stattgefunden hat. Und zwar markirt sich die Linie einmal durch die stellenweise Anläufung von Leukocyten und rothen Blutkörperchen und ferner dadureh, dass die Sklerafasern ausserhalb derselben einen normalen geraden Verlauf haben, während die nach innen zu gelegenen eine leichte wellige Schlängelung zeigen. Das hintere Ende dieser Linie im Schnitt entspricht ungefähr der Ansatzstelle des Rectus externus, an welcher eine ziemlich ansgedehnte diffuse Blutung in die oberen Skleraschichten und die hier bedeutead verdickte Episklera stattgefunden hat.

Was endlich die Lage der luxirten Linse betrifft, so liegt ihr vorderer Rand unmittelbar hinter der äusseren Oeffnung des im Schnitte sich darbietenden Wundeanals, so dass die spärlichen Sklerafasern, welche die obere Grenze desselben bilden, bis direct an die linse herantreten und sogar noch deren bindegewebige Ueberkleidung in ihrem vordersten Abschnitte bilden helfen. Makroskopisch ist die Linse etwa normaler Grösse. Die der Sklera aufliegende Hinterfläche, welche an dem Fehlen des Kapselepithels kenntlich ist, erscheint bedentend abgeflacht, fast plan; die Convexität der Vorderfläche dagegen hat in einem Grade 
zugenommen, dass sie derjenigen einer normalen Linsenhinterfläche mindestens gleich kommt.

Die Linsensubstanz lässt zum Theil den normalen Bau der Linsenfasern mit ihrer regelmässigen Anordnung deutlich erkennen, zum Theil ist sie aber körnig getrübt und von Spalten und Hohlränmen durchzogen, welche mit Schollen und Myelinkugeln erfüllt sind. Am ausgesprochensten sind diese Veränderungen, wie gewöhnlich, am Aequator, wo sich die bekannten Morgagni'schen Schollen in grosser Menge und Ausdelinung finden. Die Kapsel ist überall intact. Ihr Epithel ist gut ausgebildet, hier und da verdickt und das Bild der Kapselcataract bietend. Es lässt sich auffallend weit über den Aequator hinaus auf die hintere Kapsel verfolgen, so dass eine periphere Zone derselben noch von Epithel bekleidet erscheint, während ihre mittleren Parthieen durchweg frei daron sind.

Mit ihrer abgeplatteten Hinterfläche liegt die Linse grösstentheils der Sklera direct auf, ohne dass - wenigstens an den centralen Theilen der hinteren Kapsel - sich eine Bindegewebssehicht zwischen diese and die Sklera schöbe. In der Peripherie dagegen, und zwar ganz besonders oben und unten, findet sich hier eine Lage nengebildeten Bindegewebes, welche in centrifugaler Richtung an Mächtigkeit zunimmt und am oberen und unteren Linsenrande eine ganz beträchtliche Dicke besitzt. Bemerkenswerth ist, dass gerade in diesen peripheren Regionen, wo die Sklera verdickt ist, eine Vereinigung der Linsenhinterfläche mit ihr nicht mebr stattfindet, sondern hier ein Spaltraum auftritt, der sich um den Linsenäquator herum nach oben zil fortsetzt (s. unten), Aeusserlich bedeckt wird die Linse durch eine dünne Bindegewebsdecke (Fig. 2,e), welche deutlich zwei Schichten erkennen lässt, eine innere straffere, welche aus regelmässig angeordneten, dicht über einander liegenden Fibrillenbündeln mit spärlichen spindeligen Kernen besteht, und eine äussere mehr lockere, welche von unregelmässig verlaufenden Bỉndegewrebszügen gebildet wird, in das zahlreiche Blutgefässe und diffus vertheiltes Blut eingelagert sind. Am deutlichsten tritt diese Verschiedenheit im Bau der Schichten bei der Färbung nach v. Gieson auf, wo sie sich schon äusserlich durch den Farbencontrast markirt, indem die innere Bindegewebslage eine intensiv rothe Färbung, ähnlich der des Skleragewebes, annimmt, die äussere dagegen ganz blass bleibt. An der Bildung dieser bindegewebigsn Ueberkleidung betheiligt sich die Sklera nur in ganz geringem Maasse, 
Ein Fall von Luxation der Linse in den Tenon'schen Raum etc. 137

nämlich nur in dem vordersten Absehnitte. Im Uebrigen ist sie lediglich aus episkleralem Gewebe herrorgegangen.

Während die Linse nun, wie gesagt, mit dem Centrum ihrer Hinterfläche der Sklera zum grössten Theil fest aufliegt, und hier nur an Stellen von beschränkter Ausdehnung flache Spalträume zwischen Linse und Linsenlager sichtbar sind, ist die obere Bedeckung durch den bindegewebigen Mantel eine so lockere, dass es - ebenso wie an der Peripherie der Linsenhinterfläche - fast nirgends zum directen Contact kommt. So entsteht in den peripher gelegten Schnitten das Bild, dass das betreffende Linsenstück überhaupt nicht mit dem Linsenlager in Berührung kommt, sondern allseits durch einen breiten Zwischenraum von ihm getrennt, frei in einer Höhle zu liegen scheint.

Soweit das anatomische Bild. Es bietet zwei seltene Befunde, einmal die exceptionelle Lage des Sklerarisses und zweitens die exceptionelle Lage der Linse. Was den ersten Punkt betrifft, so möchte ich auf die gewiss interessante Frage, wie die Ruptur an dieser ganz aussergewöhnlichen Stelle zu Stande kam, nicht näher eingehen. Denn da von den mannigfachen Theorieen über den Mechanismus der Skleralruptur bisher keine völlig befriedigend und allgemein anerkannt ist, obgleich sie zum Theil auf umfangreichem Beobachtungsmaterial basiren, so erscheint es misslich, einen einzelnen Fall nach dieser Richtung hin etwa ausnützen zu wollen, zumal bei der Dürftigkeit der anamnestischen Angaben, auf deren Wichtigkeit für die Beurtheilung des Zustandekommens einer Skleralruptur neverdings Müller ${ }^{1}$ ) mit Recht hingewiesen hat. Ich beschränke mich daher auf die Besprechung der anatomischen Verhältnisse an der luxirten Linse.

Betrachten wir zunächst die bisher beobachteten Fälle von Luxation der Linse in den Tenon'schen Raum, so ist bei der Kürze der Darstellungen und dem Fehlen der histologischen Untersuchung nicht allzuviel aus ihnen zu ersehen.

1) Loc. cit. S. $2-3$. 
Wadsworth ${ }^{1}$ ) ist der erste, der über einen derartigen Fall, ganz kurz, berichtet. Wir erfahren jedoch nur, dass die Enucleation des amaurotischen Auges zwei Wochen nach erfolgtem Trauma wegen Schmerzen und Irritation des anderen Auges nothwendig wurde. Ueber die Art des Traumas, den Sitz der Ruptur, die Lage der Linse wird nichts mitgetheilt.

Montagnon ${ }^{2}$ ) enucleirte das Ange zwei Monate nach dem Trauma (Fall auf den Kopf). Auch hier bestanden Amaurose und periorbitale Schmerzen. Die $1 \mathrm{~cm}$ lange Skleralwunde befand sich "oben aussen"; nähere Angaben fehlen. Die an Volumen verminderte Linse sass hinter der oberen Uebergangsfalte zwischen M. rectus superior und externus. Aus dem verminderten Volumen lässt sich mit hoher Wahrscheinlichkeit auf eine Verletzung der Kapsel schliessen.

Bei dem von Müller ${ }^{3}$ ) beschriebenen Falle handelt es sich um einen Kuhhornstoss. Der Riss geht vom Limbus aus und reicht meridional bis zum Aequator. Die Linse liegt in der Gegend des Aequator, aber so weit nach vorn, dass sie zum Theil noeh von Conjunetiva bedeckt ist. Der vordere Rand liegt in der Verbindungslinie der Ansätze des M. rectus superior und externus, der hintere Rand im Aequator.

Die Lage der Linse ist in den beiden letzten Fällen, übereinstimmend mit unserer Beobachtung, im oberen äusseren Bulbusquadranten; bei Montagn on scheint sie sogar ebenso weit nach binten gelegen zu haben, wie dieser Fall überhaupt hinsichtlich der Localisation der Linse dem unsrigen wohl ziemlich ähnlich gewesen ist. Die Rupturstelle ist bei Montagnon ebenfalls in jenem Quadranten; leider fehlt jede Angabe darüber, wie weit nach hinten sie lag, ob also die Linse direct an ihrer Austrittsstelle liegen blieb oder noch weiter gewandert ist. Bei Müller handelt es sich um

1) Dislocation of the lens under Tenon's capsule. The americ. journ. of ophthalm. 1885. Vol. II, p. 144.

2) Luxation du cristallin. Archives d'ophthalm. 1887. Tome VII. 3. S. $204-206$.

3) Loc. cit. S. $32-33$ Fall 5; S. 34 ; S. $36-37$ Krankengeschichte. 
Ein Fall von Luxation der Linse in den Tenon'schen Raum etc. 139

einen mehr meridional verlaufenden Riss, an dessen Ende sich dann gleich die Linse anschliesst.

Da diese wenigen bisher beobachteten Fälle nur makroskopisch beschrieben sind, muss ich mich zum Vergleich der histologischen Verhältnisse an die bei subconjunctival luxirten Linsen erhobenen Befunde halten, welche ein ganz ähnliches Bild bieten und daher hier gleichfalls eine kurze Besprechung erfahren mögen.

Wenn man ron den durch Incision in die Conjunctiva erhaltenen Linsen absieht, wie sie von A. v. Graefe ${ }^{1}$ ), Tornatola ${ }^{2}$ ) u. A. beschrieben sind, und welche in jenem Falle das Bild einer "gewöhnlichen Cataract", in diesem das einer "beginnenden Corticalcataract" darbot, so habe ich nur sechs Fälle in der Literatur auffinden können, in denen der enucleirte Bulbus, mehr oder weniger genau, untersucht wurde.

$\left.\Delta l t^{3}\right)$ findet in seinem Falle, von dem eine Krankengeschichte leider nicht vorhanden war, Folgendes: Conjunctiva über der Linse in derbes, pigmenthaltiges stark vascularisirtes Bindegewebe verwandelt und fest mit der Linsenkapsel verlöthet. Letztere verdickt, stark gefaltet und zerrissen. Normales Kapselepithel fehlt. Unter der Kapsel ein corneaähnliches Spindelgewebe mit grossen Rundzellen. Linsengewebe von normaler Structur, aber kömig getrübt. Ueberall Morgag'ni'sche Flüssigkeiten zwischen den Fasern.

In dem Falle von Riegel $\left.{ }^{\Delta}\right)$ ist die Conjunctiva stellenweise mit der Linsenkapsel durch straffes gefässreiches Bindegewebe verwachsen. Kapsel intact, aber merkwürdiger Weise auch in ihrem hinteren Abschnitte mit Epithel

1) Fälle vọn Linsendislocationen. v, Graefe's Arch. f. Ophthalm. 1854. Bd. I, 1. S. 336-337.

2) Contribuzione allo studio della lussazione del cristallino sotto la congiuntiva. Bolletino d'oculistica. 1888. Anno X, p. 81-83.

3) Ueber einen Fall von subconjunctival eingeheilter Linse. Archiv f. Augen- und Ohrenheilkunde. 1877. Bd. VI, S. 8-12.

4) Zur Pathologie der subconjunctivalen Linsenluxation. Inaug.Dissert. München 1888. 
(Pseudoepithel) ausgekleidet; nur an einer Stelle der Vorderkapsel fehlt das Epithel. Stellenweise Kapseleataract. Linse selbst cataractös; die stärksten Veränderungen im Aequator. Enucleation fünf Wochen nach erfolgtem Trauma.

Falchi ${ }^{1}$ ) hat zwei Fälle untersucht; leider war mir seine Arbeit nicht zugänglich. Aus dem Referat in Nagel's Jahresberichten und den von Riegel ${ }^{2}$ ) angeführten Citaten entnehme ich Folgendes: Fall I: Enucleation 21 Tage nach der Verletzung. Conjunctiva stellenweise mit der Linsenkapsel ver1öthet, welche nur an diesen Stellen normales Epithel zeigt. Kapsel zerrissen und durch eingedrungenes Blat von den Linsenfasern getrennt. Fall II: Enucleation ein Jahr nach der Verletzung. Kapsel geborsten, innerhalb derselben reichliche Neubildung von Bindegewebe. Kapselepithel fehlt vollständig.

Müller, der die genaueste Beschreibung: der subconjunctival luxirten Linse giebt, hat ebenfalls zwei Fälle untersucht. Fall $\mathrm{I}^{3}$ ): Enucleation $4 \frac{1}{2}$ Wochen nach dem Trauma. Linse cataractös, doch ohne Myelinkugeln. Corticalis verflüssigt, zwischen ilhr und Kapsel ein Hohlraum. In der hinteren Kapsel ein Riss, dessen Enden vielfach eingerollt sind. Vordere Kapsel mit normalem Epithel ausgekleidet und durch eine Schichte Blut und eine nicht continuirliche Lage Fibrin mit dem Linsenlager verbunden. Wo die Kapsel fehlt, straffes kernarmes Bindegewebe zwischen Linsenmassen und Umgebung. Fall $\left.\mathrm{II}^{4}\right)$ : Enucleation einige Wochen nach erfolgter Verletzung. Kapsel hängt mit der Umgebung fest zusammen; Verbindung durch straffes Bindegewebe hergestellt. Kapsel intact; ihre epithellosen Theile mit Rundzellen durchsetzt.

Stellen wir diesen Befunden unsere bei Luxation der Linse in den Tenon'schen Raum gefundenen Resultate gegenüber, so finden wir, dass die Veränderungen, welche die Linse selbst eingegangen ist, sowohl als diejenigen,

1) Sulla lussazione del cristallino sotto la congiuntiva. Annali d'ottalmol. XIV. Referat: Nagel's Jahresberichte 1886. XVI, S. 209.

2) Loc. cit. S. 18-19.

3) Loc. cit. S. 32-33 Fall 2 der Statistik; S. 35-36 Krankengeschichte; S. 47-57 anatomischer Befund.

4) Loc. cit. S. $32-33$ Fall 7 der Statistik; S. 37 Krankengeschichte; S. 59-62 anatomischer Befund. 
Ein Fall von Luxation der Linse in den Tenon'schen Raum etc. 141

welche sie in ihrer Umgebung hervorgerufen hat, verhältnissmässig sehr gering sind, obwohl die Zeit, die zwischen Trauma und Enucleation liegt, - abgesehen ron dem zweiten Falle von Falchi - hier nicht kürzer war als dort. So ist die Linsenkapsel völlig intact geblieben, ein unter den sechs Fällen nur zweimal beobachtetes Verhalten. Das Kapselepithel ist normal und wohlausgebildet, was bei den meisten subconjunctivalen Linsen nicht der Fall war, und greift auffallend weit auf die Hinterfläche der Linse über, ohne dieselbe indessen in Form eines "Pseudoepithels" in ganzer Ausdehnung zu bekleiden, wie das Riegel ${ }^{1}$ ) beschrieben hat. Ganz besonders auffallend jedoch ist die geringe Tendenz der Linse zur entzindlichen Verklebung mit ihrer Umgebung. Denn während in allen erwähnten Fällen von subconjunctivaler Linsenluxation ausnahmslos hervorgehoben wird, dass die Conjunctiva, sei es vollständig, sei es theilweise, fest mit der Linsenkapsel verlöthet war, ist in unserem Falle von einer analogen Vereinigung der Linse mit dem sie bedeckenden episkleralen Gewebe, mit der Tenon'schen Kapsel, nichts nachzuweisen. Die einzige innigere Berührung zwischen Linse und Linsenlager findet an der hinteren Kapsel statt, welche mit dem grössten Theil ihrer centralen Parthieen der Sklera direct aufliegt. Dass es aber auch hier nicht zu einer entzündlichen Verlöthung gekommen ist, geht einmal daraus hervor, dass sich auch hier noch stellenweise flache Hohlräume zwischen Sklera und Linsenkapsel finden, und ferner daraus, dass gerade an diesen Stellen eine Schicht neugebildeten Bindegewebes von nennenswerther Mächtigkeit nicht zu. constatiren ist, während gerade in der Peripherie der Linsenfläche, wo kein innigerer Contact zwischen Linse und Sklera besteht, sich eine Bindegewebslage von ansehnlicher Dicke entwickelt hat.

1) Loc. cit. S. 19. 
Der Grund für diese eigenthümlichen Verhältnisse liegt meines Erachtens darin: Die Linse ist in ihrer neuen, durch die Luxation geschaffenen Lage fest auf die Sklera gedrïckt; nicht durch die Spannung der abgehobenen Kapsel, denn diese bildet eine so weite, geräumige Umhïllung, dass sie an verschiedenen Stellen noch in Falten liegt, sondern durch das umgebende orbitale Fettgewebe. In Folge dieses Druckes ist es zu einer Abflachung der Hinterfläche der Linse gekommen, welche in der resistenten Sklera ein einigermaassen hartes Widerlager fand, während die Vorderfläche eine vicariirende Zunahme ihrer Convexität erfuhr. Intra vitam muss natürlich die Tenon'sche Kapsel gleichfalls fest auf die Linse gepresst gewesen sein, hat sich aber post enucleationem, da sie mit der Linse nicht fest verlöthet war, wieder von ihr abgehoben.

Die Linse wirkte in ihrer newen Lage als Fremdkörper; sie musste als solcher eine mechanische Reizung ausüben und zu einer Wucherung des umgebenden Gewebes führen, welche bei glatten unlöslichen Körpern bekanntlich sehr geringfügig ist. Dass diese Wucherung gerade an der Stelle der innigsten Berïhrung der Linse mit ihrem Lager, d. h. im Centrum der hinteren Kapsel, am geringfügigsten ist, liegt offenbar daran, dass der hohe Druck, den die sich abflachende Wölbung der Linsenhinterfläche hier ausübte, einer Proliferation des angrenzenden Gewebes hinderlich war, während der geringere Druck in der Peripherie eine lebhaftere Gewebsneubildung gestattete. Die bedeckende 'Tenon'sche Kapsel, welche vom orbitalen Zellgewebe gleichmässig der Linsenoberfläche angedrückt wurde, antwortete auf den mechanischen Reiz mit der Bildung einer gleichmässigen dünnen Lage strafferen Bindegewebes. 
Ein Fall von Luxation der Linse in den Tenon'schen Raum etc. 143

Erklärung zu den Abbildungen auf Tafel VI. Fig. 1 und 2.

Fig. 1. Uebersichtsbild nach einem etwa durch die Mitte der Linse gehenden Schnitte.

Fig. 2. Oberhalb des Linsencentrums gelegener Schnitt durch Skleralriss und Linse.

$$
\begin{aligned}
c . & =\text { Cornea } \\
i . & =\text { Iris. } \\
s . & =\text { Sklera, } \\
a . & =\text { vordere Wundlippe. } \\
b . & =\text { hintere Wundlippe. } \\
n . & =\text { Narbengewebe. } \\
r . & =\text { Retina. } \\
c h . & =\text { abgehobene Chorioidea. } \\
l . & =\text { luxirte Linse. } \\
e . & =\text { Episklera, die Linse bedeckend. }
\end{aligned}
$$



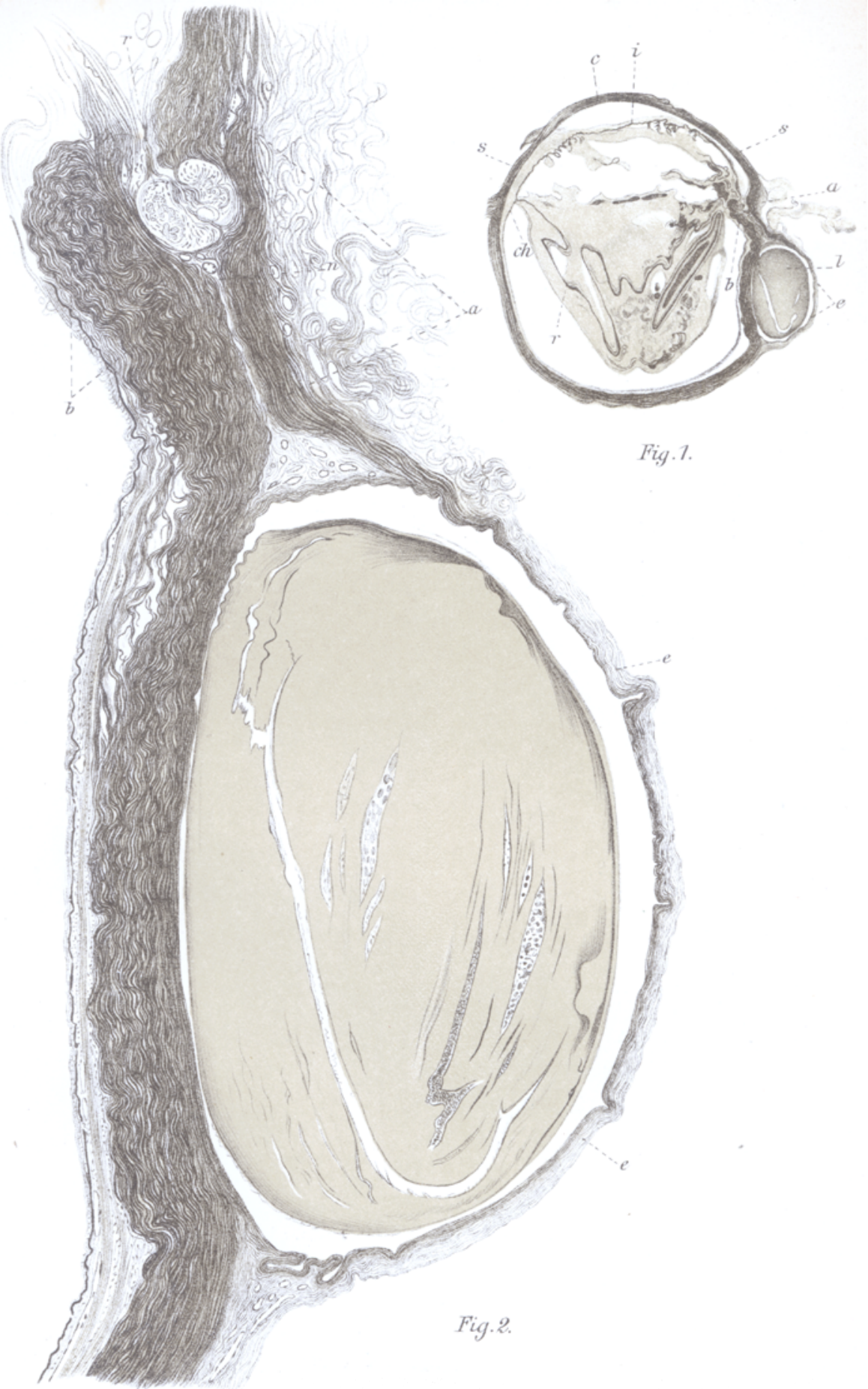